\title{
Prevalencia de Traumatismos por Accidentes de Motocicleta en Salas de Internación del Centro de Emergencias Médicas: Periodo Octubre a Diciembre del 2010
}

\author{
Alfonso Peralta, Alejandra Soto, Milena Soto, Cesar Tintel, Gustavo Machain Vega
}

\section{RESUMEN}

Los traumatismos por motocicletas presentan una prevalencia en aumento en muchos países.

Objetivo: Poder determinar la prevalencia de traumatismos de motocicletas en un periodo definido y en centro hospitalario de referencia del trauma.

Materiales y métodos: Estudio observacional descriptivo retrospectivo de corte transversal. Muestreo No probabilístico de casos consecutivos, en un periodo de tres meses consecutivos.

Resultados: Presentamos 1803 pacientes internados, de los cuales 964 eran varones $(53,5 \%)$ y 839 eran mujeres $(46,5 \%)$. La prevalencia de pacientes internados que sufrieron traumatismos por accidente de moto corresponden al $23,6 \%$ (426 pacientes); entre ellos 353 eran varones $(82,8 \%)$ y 73 eran mujeres (17,2\%). La media de internación fue entre 2 a 5 días en 143 casos $(33,6 \%)$ Con respecto a la procedencia, 87 eran de Asunción (20,5\%) y 339 del interior del país $(79,5 \%)$. La prevalencia de traumatismo craneoencefálico en ésta serie es de $43,9 \%$ y la de politraumatizados 33\% La prevalencia de óbitos es de 4,7\% (n: 20$)$ recibiendo el alta el 95,3\% (n: 406).

Conclusiones: La prevalencia de accidentes de motocicletas en la Sala de Internación es importante e involucra generalmente varones en especial. Es recomendable fijar pautas de control para prevención de esta forma de trauma.

Palabras clave: Motocicletas, Traumatismos craneoencefálicos, Interior del país.

\section{ABSTRACT \\ Motorcycle injuries represent an increased prevalence in many countries.}

Objective: To determine the prevalence of motorcycle injuries in a defined period and in a reference trauma hospital.

Materials and methods: Observational, descriptive, transverse, retrospective study. Nonprobabilistic sampling of consecutive cases over a period of 3 months.

Results: Total of 1,803 admitted patients, of which 964 were males $(53.5 \%)$ and 839 were women $(46.5 \%)$. The prevalence of hospitalized patients who suffered injuries from motorcycle accident corresponded to $23.6 \%$ (426 patients) 353 were males $(82.8 \%)$ and 73 were women $(17.2 \%)$. The average hospital stay was between 2 to 5 days of the 143 cases (33.6\%). Regarding the origin, 87 were from Asuncion (20.5\%) and 339 in rural provinces $(79.5 \%)$. The prevalence of head trauma in this series is 43.9 and $33 \%$ of multiple trauma. The prevalence of death is of $4.7 \%(n=20)$ and $95.3 \%(n=406)$ were discharged.

Conclusion: The prevalence of motorcycle accidents in the ward is important and usually involves males. We recommend setting monitoring for prevention of this type of trauma.
Keywords: Motorcycles, Head trauma, Rural provinces.

How to cite this article: Peralta A, Soto A, Soto M, Tintel C, Vega GM. Prevalencia de Traumatismos por Accidentes de Motocicleta en Salas de Internación del Centro de E mergencias Médicas: Periodo Octubre a Diciembre del 2010. Panam J Trauma Critical Care Emerg Surg 2012;1(2):122-126.

\section{Source of support: Nil}

\section{Conflict of interest: None}

\section{PREGUNTA DE INVESTIGACIÓN}

Cuál fue la prevalencia de traumatismos por accidentes de motocicleta en las salas de internación del Centro de Emergencias M edicas durante los meses de octubre, noviembre y diciembre del año 2010?

\section{OBJETIVO GENERAL}

Determinar la prevalencia de traumatismos por accidentes de motocicleta en salas de internación del Centro de Emergencias M édicas.

\section{OBJETIVOS ESPECÍFICOS}

- Establecer la prevalencia de traumatismos según sexo y lugar del accidente.

- Indicar el tiempo promedio de internación por traumatismo sufrido en un accidente de motocicleta.

- Determinar la prevalencia de traumatismo craneoencefálico entre los pacientes traumatizados por accidente de motocicleta.

- Establecer la prevalencia de óbitos por complicaciones de traumatismos por accidentes de motocicleta.

\section{JUSTIFICACIÓN}

En los últimos diez años la cantidad de motocicletas que circulan por las calles del país se ha multiplicado varias veces, según fuentes periodísticas solo entre enero y abril de 2010 han sido adquiridas 850 mil motocicletas en distribuidores legales, consideramos que las grandes facilidades para la adquisición de los vehícul os sumado a una mala formación ciudadana y víal han hecho que los accidentes que involucran a motociclistas se vuelvan al go totalmente rutinario y un verdadero problema de salud pública que requiere la utilización de gran cantidad de 
Prevalencia de Traumatismos por Accidentes de Motocicleta en Salas de Internación del Centro de Emergencias Médicas

recursos humanos y financieros; es por ello una cuestión interesantísima observar en qué grado los accidentes moto ciclísticos ocupan a un centro de referencia de trauma como lo es el Centro de Emergencias M édicas 'Prof Dr M anuel Giagni'.

\section{INTRODUCCIÓN}

El Centro de E mergencias M édicas se ve casi todos los fines de semana rebasado en su capacidad debido al consumo de bebidas al cohólicas y de la anarquía en las calles y rutas del país para cuya comprobación basta salir de vez en cuando. Solo en el 2008 se reportaron 1200 muertes en las rutas , 50\% de las cuales fueron debido a accidentes en motos. ${ }^{5} \mathrm{E}$ el año $2010,66 \%$ de los accidentados en ruta atendidos en el Centro de $E$ mergencias M édicas tuvieron una moto involucrada en el percance. ${ }^{6}$ y basta realizar un día de guardia en la urgencia para ver casi toda las modal idades de accidentes en moto: caídas, choques con peatones, choques con columnas, árboles, automóviles y con otras motos, muchas veces sin casco, otras veces sin luz y otras alcoholizados. Al igual que en la urgencia en la sala de internados dominan los 'motoqueiros' con diversas lesiones que incluyen graves traumatismos craneoencefálicos que tienen sometido al paciente a la cama por periodos prolongados, pacientes quirúrgicos infectados y por supuesto politraumatizados, por tanto es imaginable el al to costo que demanda al estado y la gran cantidad de familias que se ven afectadas.

\section{Marco Teórico}

El trauma es definido como lesión orgánica y psíquica causada por situaciones de alteración del entorno de un individuo. En general, ocurre cuando hay una disrupción súbita de la relación estable entre ese individuo y su medio; en él intervienen fuerzas violentas de índole física, química o psíquica y todas estas intervenciones tienen como consecuencia efectos tanto físicos como psíquicos. ${ }^{4}$ El concepto de politraumatizado implica la presencia de dos 0 más lesiones traumáticas que ponen en peligro la supervivencia, habitualmente un TCE de gravedad moderada o grave combinada con alguna otra lesión, - lesiones múltiples que se acompañen de pérdida de cantidades masivas de sangre que pueden Ilevar a un shock hipovolémico. ${ }^{9}$

En el ámbito mundial se reporta anualmente un cuarto de millón de fallecidos por traumatismos en accidentes de tránsito, ocurriendo una muerte cada 50 segundos y un traumatismo cada 2. Cada día mueren en el mundo más de 3000 personas por lesiones resultantes del tránsito. En los países de ingresos bajos y medianos se concentra aproximadamente un $85 \%$ de esas muertes y el $90 \%$ de la cifra anual de años de vida ajustados en función de la discapacidad (AVA D) perdidos por causa de esas lesiones. ${ }^{8}$ Se calcula además que se producen de 100-200 lesionados por cada fallecido por accidentes, y hay autores que plantean que en el niño pueden llegar a 1000. Estas lesiones por accidentes responden al $15 \%$ de la carga de muerte e invalidez, y se pronostica que puede llegar hasta el $20 \%$ en el 2020. ${ }^{3}$ En todo el planeta muere cerca de 1 millón de personas al año en accidentes del tránsito y entre 10 y 15 millones sufren lesiones por esta causa. Portugal tiene la tasa de mortalidad más al ta de accidentes por vehículos a motor del mundo. Las tasas de colisiones y de mortalidad por este tipo de accidentes muestran una relación directa con la densi dad del tráfico. ${ }^{2}$ L os accidentes de tránsito constituyen en el mundo, entre la tercera y cuarta causa de muerte para todas las edades, pero es la primera por debajo de los 45 años de edad. L a pérdida de la vida y las incapacidades transitorias o permanentes crean un costo económico incalculable, y el impacto familiar y social produce dramáticas consecuencias en los países desarrollados y en los que están en vías de desarrollo. Estos costos no son sólo derivados de la atención a las víctimas por parte de las instituciones, equipo de salud y planes de seguridad, sino también a los daños a la propiedad y pérdida de salarios-producción, discapacidades transitorias o permanentes, alejamiento del entorno sociallaboral; creando una dependencia familiar e institucional. En los Estados U nidos deA mérica, según datos del 'Comité de Trauma del Colegio de Cirujanos', ocurren 150.000 muertes al año, que llegan a triplicar el número de lesionados con diversidad de incapacidades. ${ }^{1}$

En Paraguay, según estudios de la Organización M undial de la Salud (OM S), el porcentaje de fallecidos, dentro del grupo de peatones, ciclistas y motociclistas, es superior al $40 \%$. Registros del M inisterio de Salud Pública y B ienestar Social, en el año 2008 , muestran que un total de 1.180 personas fallecieron a causa de accidentes de tránsito y en el 2009, otros 1.163. Los mayores porcentajes de estos fallecimientos se registran en peatones y en conductores de motocicletas con 32,5 y $42,7 \%$ respectivamente, representando un total de $75,2 \%$ como causa de muerte ${ }^{7}$ Según Vigilancia de la Salud, esto nos sitúa dentro de la franja de países con elevadas tasas de fallecidos por accidentes de tránsito, con 17,1\% por cada cien mil habitantes, además de conllevar un gran costo para el sector salud y el sector asegurador, con gran impacto social, ya que las pérdidas en capital social son incal culables; por lo que sostienen es urgente enfrentar este problema, con la participación del gobierno, del sector privado y especialmente, con los usuarios de las vías. Solo en el servicio de urgencias del Centro de Emergencias M édicas en el 2010, según su oficina de estadísticas, fueron atendidos 6,854 personas por accidentes de motocicleta de 
los 10.261que Ilegaron al hospital por algún accidente de tránsito. ${ }^{6}$

\section{MATERIALES Y MÉTODOS}

Diseño: Estudio observacional descriptivo retrospectivo de corte transversal.

Estadística: Tablas y gráficos estadísticos elaborados con Microsoft Excel 2007. Se recurrirá al uso de estadística descriptiva.

Variables:

- Sexo

- Lugar del accidente

- Días de Internación

- Politraumatismos

- Traumatismo craneoencefálico.

Materiales:

- Planillas de internación del Centro de Emergencias M édicas.

- Formulario de recolección de datos.

\section{Sujetos en Estudio}

Población enfocada: Pacientes internados en sal as del Centro de Emergencias M édicas.

Población accesible: Pacientes internados en el Centro de E mergencias M édicas en el periodo que comprende a los meses de octubre, noviembre y diciembre del 2010.

\section{Criterios de Inclusión}

Pacientes internados en el Centro de Emergencias M édicas en el periodo que comprende a los meses de octubre, noviembre y diciembre del 2010.

\section{Criterios de Exclusión}

Pacientes traumatizados en accidentes de motocicleta cuyos datos no estén completos en la planilla de internación.

Reclutamiento: Se identificará a los pacientes internados por traumatismo sufrido en un accidente de motocicleta y a los internados por una causa distinta, se utilizaran las planillas de internación para hallar la prevalencia de accidentados en moto que se encuentran internados de acuerdo a los datos en ellas disponibles.

Muestreo: No probabilístico de casos consecutivos.

\section{Consideraciones Éticas}

- Se mantuvo la confidencialidad de los pacientes

- Consentimiento informado del Hospital al momento de la recolección de $D$ atos

- Los resultados serán expuestos para beneficio de los pacientes afectados.

\section{ANÁLISIS DE DATOS}

\section{Resultados}

La muestra consta de 1803 pacientes internados, de los cuales 964 eran varones $(53,5 \%$ ) y 839 eran mujeres $(46,5 \%$ ). La prevalencia de pacientes internados que sufrieron traumatismos por accidente de moto corresponden al 23,6\% (406 pacientes) (Figura 1); entre ellos 353 eran varones $(82,8 \%$ ) y 73 eran mujeres $(17,2 \%)$ (Figura 2$)$. Con respecto a la procedencia, 87 eran de A sunción $(20,5 \%$ ) y 339 del interior (79,5\%) (Figura 3).

El tiempo de internación desde el momento del accidente va desde 1 a 87 días, 31 (7,3\%) se internaron por 1 día, 143 (33,6\%) entre 2 a 5 días, $125(29,3 \%)$ de 6 a 10 días y 127 $(29,8 \%)$ por más de 10 días (Figura 4).

Considerando el traumatismo craneoencefálico entre los pacientes traumatizados por accidente de motocicleta, la prevalencia es de 43,9\% (Figura 5). Los pacientes politraumatizados corresponden al 33\% (141 pacientes) (Figura 6).

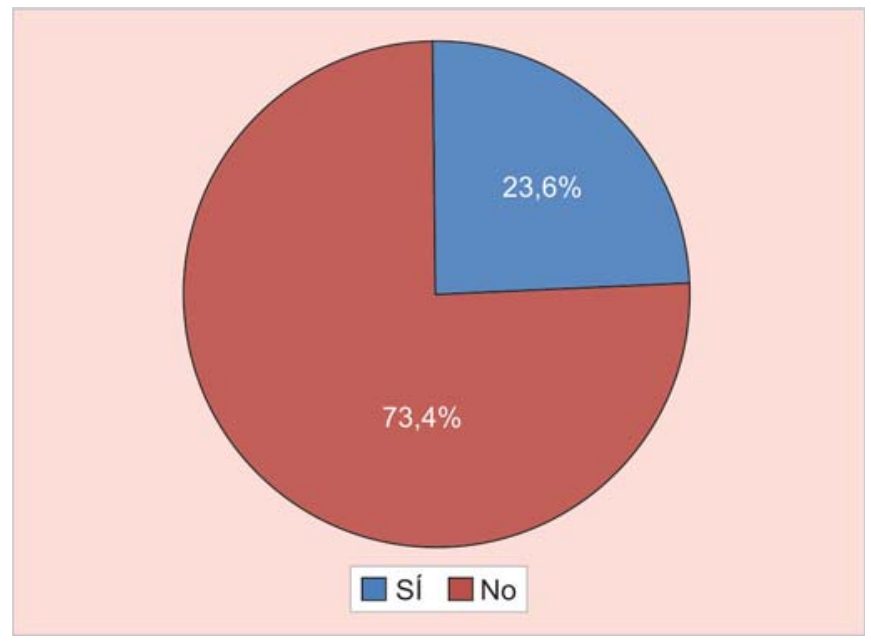

Figura 1: Prevalencia de traumatismos em accidentes de motocicleta

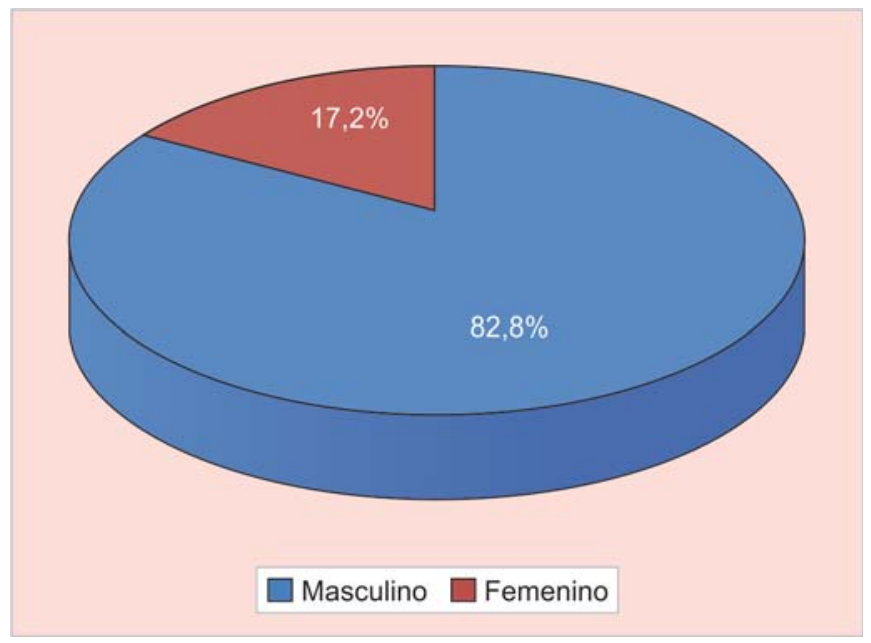

Figura 2: Sexo 
Prevalencia de Traumatismos por Accidentes de Motocicleta en Salas de Internación del Centro de Emergencias Médicas

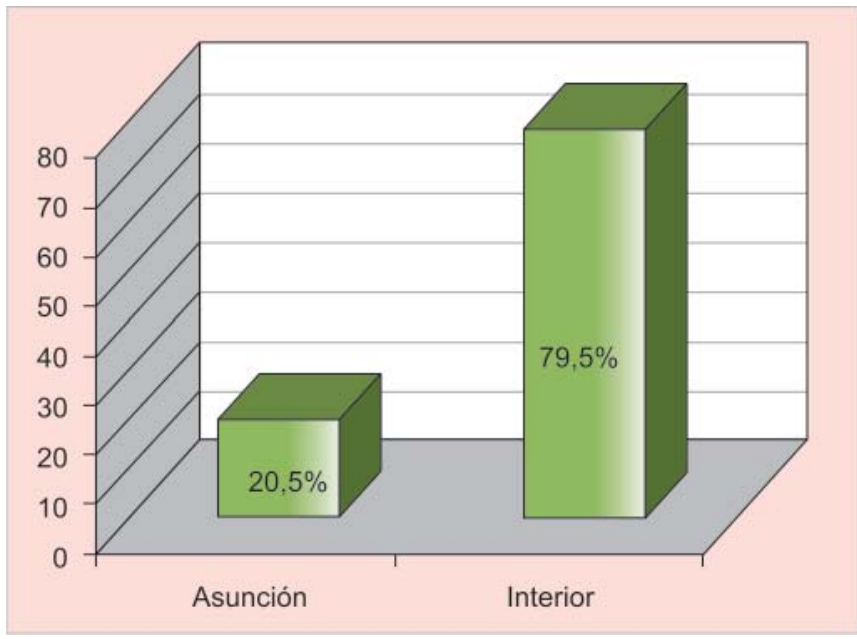

Figura 3: Procedencia

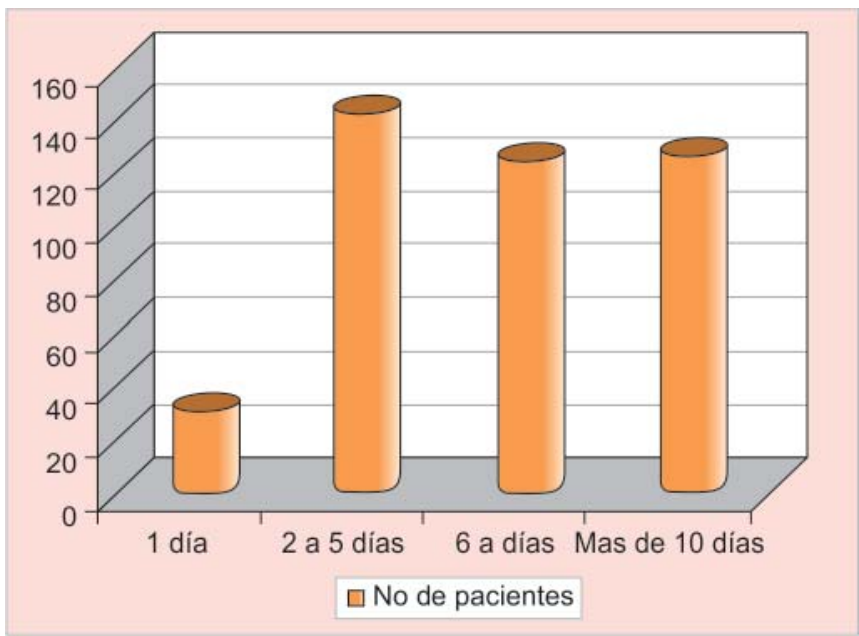

Figura 4: Días de internación

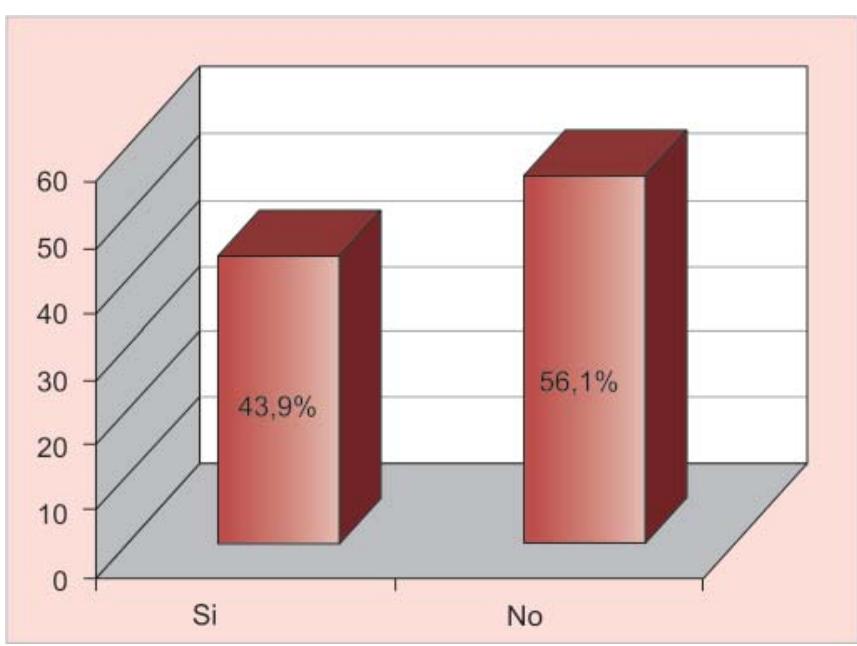

Figura 5: Traumatismo craneoencefálico

La prevalencia de óbitos debido a complicaciones es de 4,7\% (20 pacientes) recibiendo el al ta el 95,3\%(406 pacientes) (Figura 7).

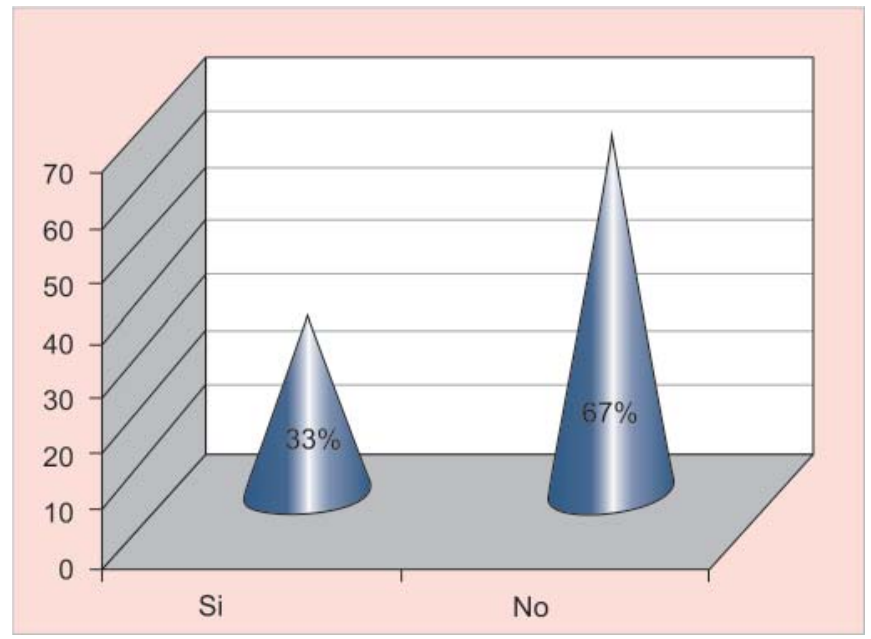

Figura 6: Politraumatizados

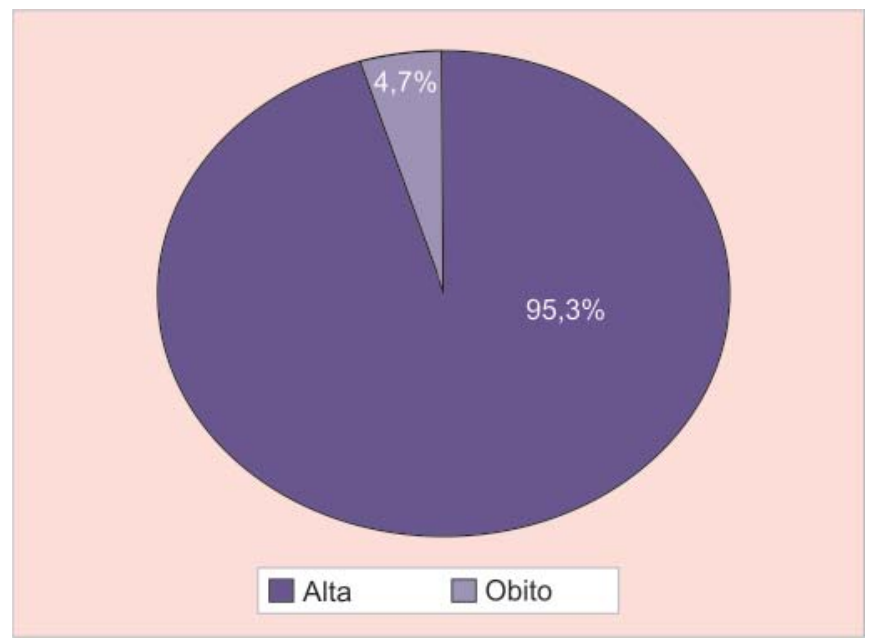

Figura 7: Desenlace

\section{CONCLUSIÓN}

En un centro de referencia para trauma como lo es el CEM los pacientes accidentados en percances de motocicleta ocupan un porcentaje importante de las camas $(23,6 \%$ en nuestro trabajo), la mayoría de ellos varones ( $82.8 \%$ del total) traumatizados por accidentes ocurridos en el interior del país (79,5\%), entre los cuales el $92.3 \%$ requirió más de un día de internación, y $29.8 \%$ más de 10 días, la mayor parte de ellos $(33,6 \%)$ permanecieron internados entre 2 a 5 días. La preval encia de TCE hallada entre estos pacientes fue del $43.9 \%$, la de politraumatizados 33\% y la de óbitos del $4.7 \%$.

\section{REFERENCIAS}

1. Cid CA . Politrauma en A rgentina la enfermedad silenciosa de las sociedades modernas. Comité de TraumaA rgentino, 2007.http:// www.samct.org.ar/comites/ortoped/politra.doc

2. M edina $E, K$ aempffer AM. Consideraciones epidemiológicas sobre los traumatismos. Rev Chilena de Cirugía. [serie en Internet] 2007. http://www.scielo.cl/pdf/rchcir/v59n3/art03.pdf 
3. Pérez GR, M artínez V M, M onteagudo NC, Tenorio CS, Perera DM , García BE, et al. Traumatismos. En: López CA , G arcia BE, Perera DM , Armas AF, Expósito CA, Peralta M M , et al (Eds). Terapia Intensiva. 2da ed. La Habana-Cuba: Editorial Ciencias M édicas; 2006.pag. 5523-761.

4. Ferraina P, Oria A . Cirugía de M ichans. Q uinta edición. Buenos A ires. Editorial El A teneo 1997.

5. B ogado L, M achain $G$, A ucejo $M$. Temas de Técnica Q uirúrgica. A sunción-Paraguay. EFACIM 2009. p 515.

6. Centro de Emergencias M édicas. Sitio Web http://www emergenciasmedicas.gov.py/interna_estadisticas.php

7. Organización M undial de la Salud. Estrategias de cooperación técnica OM S-República del Paraguay. Washington DC 2010 http://www.who.int/countryfocus/cooperation_strategy/ccs_pry es.pdf

8. M urray $C J L$, Lopez $A D$, eds. The global burden of disease: A comprehensive assessment of mortality and disability from diseases, injuries, and risk factors in 1990 and projected to 2020. B oston, M A , H arvard School of Public H ealth, 1996 http://www. who.int/violence_injury_prevention/publications/road traffic/ world report/summary_es.pdf

9. Villalba AJ, M orales R. CIRU GIA. Fundamentos y terapéutica Efacim 2001;pág 215.

\section{ACERCA DE LOS AUTORES}

\section{Alfonso Peralta}

Estudiante de $\mathrm{M}$ edicina $5^{\circ}$ Curso, $\mathrm{F}$ acultad de Ciencias M edicas FCM , Universidad $\mathrm{N}$ acional de A suncion, UNA

\section{Alejandra Soto}

Estudiante de $\mathrm{M}$ edicina $5^{\circ} \mathrm{C}$ urso, $\mathrm{F}$ acultad de Ciencias M edicas FCM , Universidad Nacional de A suncion, UNA

\section{Milena Soto}

Estudiante de $M$ edicina $5^{\circ}$ Curso, $\mathrm{F}$ acultad de Ciencias M edicas FCM , Universidad Nacional de A suncion, UNA

\section{Cesar Tintel}

Estudiante de M edicina $5^{\circ}$ Curso, F acultad de Ciencias M edicas FCM Universidad Nacional de A suncion, UNA

\section{Gustavo Machain Vega (Correspondencia)}

Professor, J efe de Catedra Tecnica Quirurgica, FCM , UN A Comision de Docencia Centro de Emergencias M edicas, Prof M anuel Giagni, A suncion, Paraguay, e-mail: gmmachain@yahoo.com 